Ungelöste Probleme von Strategien für einen umweltverträglichen Industriewandel

\section{Politikexperimente mit ungewissem Ausgang}

\author{
Auf den ersten Blick scheint Transition Management eine ideale Lösung für \\ offensichtliche und schleichende Umweltprobleme. Der Ansatz braucht zwar \\ etwas Zeit, lockt aber mit der Möglichkeit für Konsensbildung, Raum \\ für Experimente und dauerhafte Beseitigung von Umweltproblemen durch \\ umfassenden technologischen Wandel. Ein genauer Blick auf das \\ vermeintliche Wundermittel offenbart allerdings eine Reihe von \\ Schwächen und ungeklärten Fragen.
}

$\mathrm{T}$ Von Klaus Jacob rotz einiger umweltpolitischer Anstrengungen zur Verbesserung der Umweltperformanz von Industrien seit der Entstehung moderner Umweltpolitik vor circa 30 Jahren, ist die industrielle Produktion in vielen Bereichen weit von einer nachhaltig umweltverträglichen Produktionsweise entfernt. Während bei einigen Schadstofftypen eine Entkopplung von Umweltnutzung und wirtschaftlichem Wachstum erreicht wurde, bleiben viele Umweltprobleme ungelöst und immer neue werden sichtbar. Die ständige Strapazierung der Natur macht einen umfassenden industriellen Strukturwandel notwendig. Zur Herbeiführung des Wandels reichen Marktmechanismen allein aber nicht aus. Der räumliche und zeitliche Horizont der Marktteilnehmer greift zu kurz, um die bei der Güterproduktion entstehenden Umweltschäden in angemessenen Preisen zu berücksichtigen. Aber auch die bisherigen politischen Anstrengungen haben die Umwelt nur unzureichend geschützt. Die staatliche Steuerung eines umfassenden Strukturwandels als eine Aufgabe an sich, ist innerhalb des politischen Systems umstritten. Diese Aufgabe steht zumindest in kurzfristiger Perspektive in einem Zielkonflikt mit der Sicherung von Arbeitsplätzen und wirtschaftlicher Entwicklung.

In den Niederlanden wird unter dem Titel Transition Management ein Steuerungskonzept entwickelt und erprobt, das die bisherigen Steuerungsdefizite zumindest teilweise ausgleichen soll (vgl. den Beitrag von Kemp in dieser Ausgabe). Das Konzept fokussiert auf Systeminnovationen, verstanden als grundlegender Wandel von technischen, sozialen, regulativen und kulturellen Regimes, die in ihrem Zusammenwirken bestimmte Bedürfnisse wie Transport, Ernährung, Behausung, Wasser oder Energie befriedigen. Ein Systemwandel erfordert die Ko-Evolution von Technologien, Infrastruktur, Regulierungen, symbolischen Bedeutungen, Wissen, industrieller Struktur, etc.. Diese Übergänge nehmen typischerweise einen Zeitraum von 30 bis 40 Jahren in Anspruch.

Der lange Zeitraum und die umfassenden notwendigen Veränderungen entziehen sich der konventionellen staatlichen Steuerung. Vertreter von Transitionsstrategien schlagen deswegen die Institutionalisierung von Transitions-Management vor, das weitergehende Steuerungsleistungen erfïllen soll. Damit wird nicht der Anspruch verbunden, Transitionen planen zu können, sondern es soll die Richtung und die Geschwindigkeit des Prozesses beeinflusst werden. Ein solches Management ist in die vier folgenden Phasen teilbar (1):

- Die Schaffung eines Innovationsnetzwerkes, einer Transitionsarena für ein definiertes Transitionsproblem mit Vertretern von Regierung, Wissenschaft, Unternehmen und NGOs. Dieses Netzwerk soll nicht mehr als zehn bis 20 Personen umfassen.

- Die Generierung von integrierten Visionen und Vorstellungen über Transitionspfade, die sich über 25 bis 50 Jahre erstrecken. Daraus sollen Zwischenziele abgeleitet werden.

- Die Durchführung von Experimenten und konzertierten Maßnahmen gemäß der Transitionsagenda. Experimente können neue Technologien, Regulierungen, Finanzierungsformen, etc. umfassen.

- Das Monitoring und die Evaluation des Prozesses sowie die Umsetzung der daraus entstehenden Lernprozesse. Erfolgreiche Experi- mente sollen schließlich im normalen Politikprozess aufgegriffen und deren Diffusion gefördert werden.

In diesem Rahmen soll eine Transitionspolitik entwickelt und umgesetzt werden, die

- Vielfalt fördert um Lock-in Situationen zu vermeiden,

- integriert und mit den konventionellen Entscheidungsmechanismen legitimiert ist,

- langfristig angelegt ist, selbst wenn keine unmittelbaren Erfolge zu verzeichnen sind,

- und mit den verschiedenen Ebenen der Politikformulierung koordiniert ist.

Transitionspolitik kann auf die vorhandenen Instrumente von Wissenschaftsförderung, Innovationspolitik, und sektoraler Politik zurückgreifen, jedoch soll ihr Einsatz koordiniert werden. Bisher gibt es keine Beispiele für Erfolg oder Misserfolg solcher Transitionspolitiken, in den Niederlanden werden solche Vorhaben erst seit 2001 implementiert.

\section{- Problemfeld Transitionsarenen}

Welche Erwartungen können mit diesen Vorschlägen verbunden werden? Sind Strategien des Transition-Managements dazu geeignet, Transitionen eine bestimmte Richtung zu geben? Die Notwendigkeit einer langfristig angelegten Politik ist angesichts der Probleme unbestritten. Zweifelhaft bleibt aber, ob und wie es gelingen sollte, aus einer kleinen Transitionsarena Ziele zu formulieren, die in der politischen Praxis Beachtung und Geltung erfahren. Dies setzt voraus, dass die dort entwickelten Visionen allgemein zustimmungsfähig werden, womit sie aber ihre steuernde Wirkung verlieren dürften. Sollten dort substantiellere Ziele formuliert werden, dann dürfte mit ähnlichen Hemmnissen wie in der Politikberatung gerechnet werden. Diskurs und Überzeugung sind zweifellos Wirkungsgrößen im politischen Prozess, aber der Modus der Interessensaushandlung wird vom Konzept der Transitionsstrategie weitgehend ignoriert; die Bedeutung von Visionen dagegen überschätzt. Die Bindungswirkung von Visionen ist gering, zudem gibt es einen Wettbewerb von verschiedenen Vorstellungen über eine wünschenswerte Zukunft. Die Nutzung von Atomenergie ist nur ein Beispiel dafür.

Zudem wird die Auswahl der Akteure für eine Transitionsarena nicht substantiiert. Sollen dort Akteure vertreten sein, die ein Interesse am Status Quo haben? Welche Motive sollen diese Akteure für ihre Beteiligung haben? Hier kann mit Blockaden gerechnet werden, wenn die Aushandlung nicht zu 
Lasten der Substanz der Ziele erfolgt. Die Kooperation in einer Transitionsarena ist zudem nicht die einzige Möglichkeit zur Interessensdurchsetzung für betroffene Akteure, dafür stehen die bisherigen Mechanismen im politischen System nach wie vor bereit. Die Akteure verfolgen innerhalb wie außerhalb der Transitionsarena nicht notwendig Gemeinwohlinteressen; die Vorschläge für ein Transitions-Management sehen auch keine Verfahren vor, wie Gemeinwohlinteressen identifiziert werden können.

\section{- Problemfeld Politikintegration}

Der Ruf nach einer weitergehenden Politikintegration, die Industriepolitik, Wissenschaftsförderung und Technologiepolitik umfasst, ist ebenfalls nicht neu. Seit der Entstehung moderner Umweltpolitik wird dies angemahnt, aber mit nur geringem Erfolg (2). Die verschiedenen Ressorts folgen unterschiedlichen Zielen und Logiken. Die Forderung nach einer weitreichenden und dauerhaften Politikintegration ist in den meisten Fällen bisher an den institutionellen Rigiditäten des politischen Prozesses gescheitert.

Weiterhin ist die Forderung kritisch zu beurteilen, dass eine Vielfalt von Optionen im Rahmen des Experimentierens offen gehalten werden soll. Zwar ist es notwendig, dass suboptimale lock-in Situationen vermieden werden. Zudem verfügen staatliche Akteure kaum über die notwendigen Informationen, um über geeignete Technologien zu entscheiden. Das Offenhalten von Möglichkeiten ist aber mit Kosten verbunden, die - wenn es um die Entwicklung von marktreifen Technologien oder Infrastrukturen geht - beträchtlich sind.

\section{Systeminnovationen und Zeitstrategien}

Ein Fokus auf Systeminnovationen ist aber dennoch sinnvoll. Die Analyse des Zusammenspiels verschiedener Akteure und von Regulationsmustern statt des Fokus auf einzelne Akteure, Produkte und Instrumente eröffnet Perspektiven auf neue Interventionspunkte und Restriktionen (3). Auf diese Weise können die Determinanten der ökologischen Performanz in Produktketten identifiziert werden und die Akteure, bei denen Politikmaßnahmen leichter und wirkungsvoller durchzusetzen sind.

Zudem eröffnet die Ausweitung der zeitlichen Perspektive die Möglichkeit für eine strategischere Ausrichtung von Politikmaßnahmen. Auch hier können sich neue Interventionspunkte ergeben: Die Entstehung und Verbreitung von Innovationen ist ein unstetiger Prozess und Phasen des radikalen technischen Wandels wechseln sich mit Phasen des inkrementalen Wandels ab. Während der Phasen erhöhter Innovationstätigkeit können neue Technologien leichter auf den Märkten etabliert werden. Die politischen Impulse, die für die Ausbreitung von Umweltinnovationen notwendig sind, können schwächer ausfallen, als in Zeiten von relativer Stabilität technologischer Systeme (4). Solche Zeitfenster können durch die Förderung von alternativen Technologien vorbereitet werden. Allerdings lassen auch die Vorschläge für Zeitstrategien (vgl. den Beitrag von Sartorius und Zundel) offen, wie die Auswahl von Technologien substantiiert werden soll, die gefördert werden und inwieweit Zeitfenster ex-ante erkennbar sind. Dies zieht praktische Probleme nach sich: Soll die Umwelt- und Technologiepolitik jetzt auf dezentrale Brennstoffzellen war- ten, die schon verfügbaren motorgetriebenen Blockheizkraftwerke fördern oder zum Beispiel auf Mikroturbinen setzen? Das gleichzeitige Offenhalten aller Optionen dürfte kostspielig sein und ein zu langes Warten auf zukünftige Technologien angesichts der gegenwärtigen Probleme lässt das Potenzial von sofort nutzbaren Umweltinnovationen unausgeschöpft.

\section{Anmerkungen}

(1) Loorbach, D.: Transition Management. Governance for Sustainability. Paper presented at the Conference Governance and Sustainability. New challenges for the state, business and civil society. Berlin 2002; download www.ioew. de/governance

(2) Jacob, K./ Volkery, A.: Instruments for Policy Integration. Intermediate Report of the Project POINT. FFU-Report 2003-06, Berlin 2003.

(3) Jacob, K.: Governance for Industrial Transformation. The Scope of the Challenge. Paper presented at the Berlin Conference on the Human Dimension of Global Environmental Change. Governance for Industrial Transformation, Berlin 2003.

(4) Nill, J.: Instrumentation of Time Strategies for an Ecological Innovation Policy: a New Role for Subsidies? IÖW-Diskussionspapier Nr. 61/04, Berlin 2004.

\section{Der Autor}

Dr. Klaus Jacob ist Forschungsleiter der Forschungsstelle für Umweltpolitik (FFU) an der FU Berlin.

Kontakt: FFU, Ihnestr. 22, 14195 Berlin.

E-Mail: jacob@zedat.fu-berlin.de

\section{ökom verlag - Wir publizieren Zukunft}

Frithjof Hager, Werner Schenkel (Hrsg.)

\section{Schrumpfungen}

\section{Wachsen durch Wandel}

Was verbindet Siedlungsentwicklung, Männer und Bakterienkulturen? In ihrer Entwicklung lässt sich ein stetiges Auf und Ab beobachten - Ausdehnung und Schrumpfung, Wachsen und Vergehen. Dynamische Veränderungen prägen das Bild völlig unterschiedlicher Lebensbereiche. Trotzdem befürchten viele scheinbar das Schlimmste, wenn wie zur Zeit das Wirtschaftswachstum einmal stagniert. Die Beiträge in diesem Buch zeigen, wie Veränderungen und Metamorphosen unser Dasein bereichern. Sie eröffnen neue Perspektiven - auch für den "homo oeconomicus».

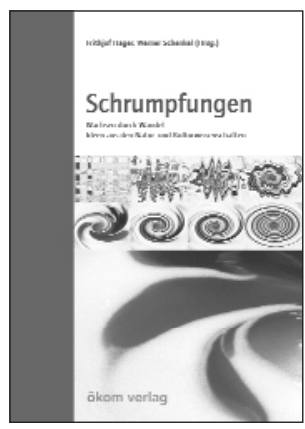


(c) 20I0 Authors; licensee IÖW and oekom verlag. This is an article distributed under the terms of the Creative Commons Attribution Non-Commercial No Derivates License (http://creativecommons.org/licenses/by-nc-nd/3.o/), which permits unrestricted use, distribution, and reproduction in any medium, provided the original work is properly cited. 